\title{
O AGENTE COMUNITÁRIO DE SAÚDE EM ANGOLA: DESAFIOS PARA SUA ATUAÇÃO E PARA A FORMAÇÃO PROFISSIONAL EM SAÚDE
}

\author{
THE COMMUNITY HEALTH WORKER IN ANGOLA: CHALLENGES TO PRIMARY HEALTH CARE \\ AND TO HEALTH PROFESSIONAL EDUCATION
}

\author{
Ana Lucia de Moura Pontes ${ }^{1}$ \\ Vera Joana Bornstein ${ }^{2}$ \\ Camila Giugliani ${ }^{3}$
}

Resumo A experiência brasileira de trabalho e formação de agentes comunitários de saúde (ACS) foi a base para a implementação do Programa de Agentes Comunitários de Saúde (Pacs) pela Direção Provincial de Saúde (DPS) de Luanda, em Angola. Esse relato reflete sobre a visita exploratória realizada, em 2009, nos municípios de Cacuaco, Cazenga, Kilamba-Kiaxi, Samba, Sambizanga e Viana, para conhecer as percepções e práticas dos ACS angolanos no que diz respeito ao seu trabalho e à saúde da população. Essa visita pretendia levantar subsídios para a discussão de cooperação técnica entre instituições brasileiras e a coordenação do Pacs da DPS, no sentido de fortalecer a formação de profissionais desse programa. Encontramos semelhanças e diferenças da experiência angolana em relação à brasileira, sendo que consideramos que os principais desafios do caso angolano são: a institucionalização do programa, a regularização do vínculo empregatício e a qualificação profissional dos agentes.

Palavras-chave atenção primária; agente comunitário de saúde; educação profissional; Angola.
Abstract The Brazilian experience of training and working with Community Health Workers was the basis for the implementation of the Community Health Workers Program by the Provincial Health Directorate in Luanda, Angola. This article reports on an exploratory visit carried out in 2009 to the municipalities of Cacuaco, Cazenga, Kilamba-Kiaxi, Samba, Sambizanga and Viana, with a view to understanding the health perceptions and practices of community health workers in Angola. This visit aimed to gather information that would engender a discussion on technical cooperation between Brazilian institutions and the coordination of the Program of Community Health Workers to strengthen the professional education of the Program. We have found similarities and differences between the Angolan experience and the Brazilian one, and we believe that the main challenges to be faced in Angola are the institutionalization of the program, the regularization of employment relationships and the professional qualifications of the community health workers.

Keywords primary health care; health community worker; health professionals; education; Angola. 


\section{Introdução}

Dentre as recomendações da Declaração de Alma-Ata (1978) para implantação da atenção primária à saúde, está o fortalecimento de recursos comunitários, como os agentes comunitários de saúde (ACS). A experiência brasileira com um programa governamental de ACS iniciou-se no Ceará, na década de 1980, sendo implantada nacionalmente a partir de 1991. Assim, os conhecimentos e as reflexões sobre a formação e o trabalho de ACS, acumulados nesses dez anos, têm sido a base para projetos de cooperação do Brasil com outros países.

Este artigo relata uma visita exploratória realizada ao Programa de Agentes Comunitários de Saúde (Pacs) de Luanda, Angola, no período de 28 de maio a 20 de junho de 2009. Essas atividades são parte do projeto aprovado no Conselho Nacional de Desenvolvimento Científico e Tecnológico (CNPq) em dezembro de 2008 e co-financiado pela Organização Pan-Americana da Saúde (Opas), intitulado “Agente Comunitário de Saúde Angolano: análise das concepções e práticas em saúde e a formação profissional".

A equipe de trabalho reuniu pesquisadores brasileiros da Escola Politécnica de Saúde Joaquim Venâncio (EPSJV) da Fundação Oswaldo Cruz (Fiocruz), da Universidade Federal do Rio Grande do Sul (UFRGS), da Universidade Federal do Ceará (UFC) e da Escola de Formação em Saúde da Família Visconde de Sabóia e dois consultores do Fundo das Nações Unidas para a Infância (Unicef). Também estão envolvidos nas atividades dois profissionais da saúde angolanos, pertencentes à Direcção Provincial de Saúde (DPS) de Luanda e o Unicef Angola. O objetivo da visita exploratória foi levantar subsídios para a discussão de cooperação técnica entre as instituições brasileiras com a coordenação do Pacs da DPS, no sentido de fortalecer a formação de profissionais desse programa.

\section{O contexto angolano e a implantação do Pacs em Luanda}

Angola se tornou independente de Portugal em 1975. Santos (2001) destaca que o processo de unificação e organização da nação angolana tem o desafio de lidar com as heranças do capitalismo colonial e a integração das diferentes sociedades africanas. Uma guerra civil envolvendo os principais partidos políticos (MPLA, Unita e FNLA) instaurou-se logo após a independência, e o acordo de paz só veio em 2002.

Além dos efeitos perversos da guerra, incluindo a grande destruição de infraestruturas básicas, como escolas e unidades de saúde, principalmente no interior do país, Santos (2001) destaca como fatores dificultadores uma economia dependente do mundo exterior e o fato de cerca de $40 \%$ da popu- 
lação viver em pobreza absoluta e buscar sua sobrevivência no setor informal e no mercado paralelo. Porém, por Angola ser o maior produtor de petróleo da África Subsaariana, muitos investimentos e acordos comerciais têm sido feitos nesses anos de reconstrução pós-guerra civil (Santos, 2001).

Oliveira e Artmann (2009) destacam, no perfil epidemiológico angolano, altas taxas de mortalidade infantil (154 por mil nascidos vivos e 250 por mil nascidos vivos para menores de 5 anos) e mortalidade materna ( 1.500 por 100 mil nascidos vivos). As endemias principais são malária, tuberculose, HIV/Aids, tripanossomíase africana, hanseníase e esquistossomose. Os autores apontam um baixo acesso aos serviços de saúde, falta de recursos humanos (com concentração dos mesmos em Luanda), baixo investimento relacionado à saúde e uma estrutura de serviços de saúde degradada.

Em 2007, o Ministério da Saúde de Angola avaliou que apenas 30\% a $40 \%$ dos angolanos tinham acesso a serviços de saúde. Quanto ao modelo de atenção à saúde, Oliveira e Artmann (2009) apontam como características uma assistência curativa, baixo nível de descentralização das ações, escassez de serviços de saúde de nível primário e secundário e precário sistema de referência e contrarreferência. Outras dificuldades referidas para a efetividade do sistema de saúde angolano são a baixa qualificação de pessoal administrativo e técnico, problemas nas redes de transporte, comunicação e demais infraestruturas, insuficientes recursos financeiros e déficit de um sistema de informação que possa subsidiar com dados confiáveis a formulação de políticas (Oliveira e Artmann, 2009).

Em 2007, a partir da participação de grupos de consultores brasileiros, a DPS de Luanda preparou o Projecto de Implantação do Programa de Agentes Comunitários de Saúde de Luanda como uma estratégia para reduzir a mortalidade materno-infantil na província. Esse projeto se embasava nas estratégias e nos resultados obtidos com a experiência brasileira do Pacs e a Estratégia de Saúde da Família, que conseguiram, a partir da década de 1980, uma melhoria significativa dos indicadores relacionados à saúde materno-infantil.

Vários estudos vêm sustentando os importantes resultados alcançados pelo trabalho dos ACS no Brasil: Svitone et al. (2000) mostraram que o trabalho dos ACS no Nordeste brasileiro contribuiu para a diminuição da mortalidade infantil e para o aumento da cobertura vacinal nas crianças; Macinko, Guanais e Souza (2006) evidenciaram uma queda importante na mortalidade infantil (de 49,7 a 28,9 por mil) associada ao aumento da cobertura da Estratégia Saúde da Família (ESF) entre 1990 e 2002. Outro estudo de Macinko et al. (2007) mostrou que o aumento da cobertura da ESF de 1999 a 2004 esteve associado a uma redução de 0,45\% na mortalidade infantil e de $1 \%$ na mortalidade por diarreia. Ainda, Aquino, Oliveira e Barreto (2009) publicaram recentemente que a redução de mortalidade infantil 
pode chegar a $22 \%$ nos locais com maior cobertura da ESF, sendo o efeito maior onde a mortalidade infantil é mais elevada e o índice de desenvolvimento humano é menor.

Assim, em fevereiro de 2007, foi constituída uma equipe da DPS para a implementação do Pacs de Luanda, que iniciou um processo de formação de formadores de ACS. Diante das dificuldades encontradas para concretizar os objetivos do programa, dois consultores brasileiros, vinculados ao Unicef, incorporaram-se a essa equipe. Como marco inicial das atividades, organizou-se o Seminário para a Formação dos Formadores dos Agentes Comunitários de Saúde, que teve como desdobramento o início da capacitação teórica de 480 ACS para a Comuna de Kikolo, no município de Cacuaco, situado na província de Luanda. Esse município foi escolhido como prioritário na implantação do programa devido às suas condições sociossanitárias precárias.

A proposta inicial do programa era formar 3.440 ACS para atender 344 mil famílias, cada ACS sendo responsável por cem famílias, em seis municípios da província de Luanda (Cacuaco, Cazenga, Kilamba-Kiaxi, Samba, Sambizanga e Viana). Com vistas à redução da mortalidade materno-infantil, foram definidas como prioridades para a atuação do ACS: o acompanhamento das gestantes e das crianças menores de 1 ano; o incentivo ao uso de mosquiteiro pelas grávidas e crianças menores de 5 anos; o tratamento domiciliar da água; a higiene doméstica. As principais tarefas estabelecidas para os ACS foram: conhecer, cadastrar e acompanhar todas as famílias de sua microárea; conhecer bem a unidade de saúde de referência para as famílias que acompanha; distribuir mensalmente o hipoclorito a todas as famílias, orientando-as para os cuidados de higiene; identificar todas as grávidas, estimulando-as a seguir o Caderno de Consultas Pré-Natais e utilizar o mosquiteiro; estimular todas as grávidas a terem o parto assistido no Centro de Saúde; estimular todas as puérperas a irem à consulta pós-parto; identificar os óbitos maternos; identificar todas as crianças nascidas, acompanhando-as e orientando as suas mães para os cuidados de saúde, especialmente para o aleitamento materno exclusivo até os 6 meses; acompanhar todas as crianças menores de 5 anos, estimulando as mães a seguirem as orientações do Cartão de Saúde Infantil; identificar os óbitos de menores de 5 anos.

O ACS deveria ser morador das comunidades, ter idade maior de 18 anos, no mínimo oito anos de escolaridade, boa relação com a comunidade e ser indicado pelos coordenadores de bairro. ${ }^{4}$ A maioria dos ACS é homem e, frequentemente, exercem outras ocupações de trabalho ou estudo, embora existam também desempregados. Os ACS não possuem qualquer vínculo empregatício formal e recebem um pagamento, denominado subsídio, de USD 50,00 (4.000 kwanzas) mensais por uma dedicação estimada em oito horas semanais de trabalho. 
Os ACS são formados e acompanhados por coordenadores municipais de ACS, cuja escolaridade é de nível técnico, em geral na área da saúde. Esses coordenadores, formalmente vinculados às unidades de saúde, ganham um adicional de USD 150,00 (12.000 kwanzas) para esse trabalho de formação e acompanhamento do trabalho dos ACS, que requer uma carga de aproximadamente quatro horas por dia. Além dos coordenadores municipais, existem os supervisores, que são ACS com o papel de supervisionar o trabalho de um grupo de ACS.

Percebemos que existem algumas diferenças tanto no desenvolvimento quanto no andamento do Pacs nos seis municípios. A Tabela 1 apresenta os números de ACS, de supervisores e de coordenadores municipais para cada local. Cacuaco, como foi o primeiro a ter o programa implantado e foi priorizado, apresenta a melhor estruturação e avanço do programa. Outro fator para o destaque dessa localidade é a qualidade do trabalho dos coordenadores, que, diferentemente dos outros municípios, têm dedicação exclusiva ao Pacs.

Tabela 1

\begin{tabular}{lccc}
\hline Situação da implantação do Pacs nos municípios da província de Luanda, Angola, em junho de 2009 \\
\hline Município & $\begin{array}{c}\text { Número } \\
\text { de ACS }\end{array}$ & $\begin{array}{c}\text { Número de } \\
\text { supervisores }\end{array}$ & $\begin{array}{c}\text { Número de } \\
\text { coordenadores } \\
\text { municipais }\end{array}$ \\
\hline Cacuaco & 1.094 & 42 & 02 \\
Sambizanga & 487 & 0 & 06 \\
Samba & 373 & 02 & 03 \\
Kilamba-Kiaxi & 176 & 13 & 03 \\
Viana & 359 & $*$ & 04 \\
Cazenga & 640 & 21 & 03 \\
Total & 3.129 & $78^{* *}$ & 21 \\
\hline
\end{tabular}

Fonte: As autoras.

Notas: * Existe, mas não obtivemos o número; ${ }^{* *}$ Número aproximado.

Em Sambizanga, não houve a formação de supervisores. Assim, é o coordenador municipal quem faz o acompanhamento de todos os ACS do município. Samba tem um número reduzido de supervisores. Este é um município situado na área rural e existe um perfil de população envelhecida, de modo que se inclui a prioridade de acompanhamento do idoso no trabalho dos ACS. Kilamba-Kiaxi tem o menor número de ACS: 176. O município de Viana vem tendo apoio dos formadores de Cacuaco para sua implantação, 
mas permanece apresentando muitas dificuldades no processo de acompanhamento dos ACS. Cazenga é o município mais populoso da província de Luanda e conta com 640 ACS.

A equipe responsável pelo programa organizou uma metodologia para a formação dos ACS, organizada com os seguintes temas: mapeamento dos bairros e divisão das microáreas com cem famílias por cada ACS, por meio de visitas domiciliares e produção de mapas; conhecimento das unidades de saúde de referência para as famílias das microáreas; cadastramento das famílias, levantamento sociodemográfico e sanitário, com sistematização e tabulação de dados; e acompanhamento e orientação de gestantes e de crianças menores de 5 anos.

Foi elaborado um conjunto de fichas para acompanhamento da cobertura e da produtividade do trabalho dos ACS. Os dados priorizados são sobre o tratamento da água, uso de mosquiteiros pelas gestantes e crianças de 0 a 5 anos, adesão ao pré-natal e consulta pós-parto, proporção de partos institucionais, proporção de crianças de 0 a 6 meses em aleitamento materno exclusivo, adesão ao calendário vacinal das crianças de 0 a 1 ano, frequência de pesagens das crianças de 0 a 5 anos, detecção de crianças com baixo peso e taxas de mortalidade materna e infantil.

As fichas, no entanto, são preenchidas de forma irregular e seus dados raramente são sistematizados. O fluxo desse sistema se organiza de modo que as fichas preenchidas manualmente pelos ACS sejam analisadas mensalmente pelos coordenadores municipais, que as repassariam para a coordenação provincial do programa, com o objetivo de avaliar o trabalho do ACS e o cumprimento das metas do programa. Como obstáculos ao bom fluxo dos dados, podemos considerar a falta de um sistema de informação informatizado, as dificuldades de comunicação entre os níveis dentro do fluxo de dados (ACS, supervisores e coordenadores nos diferentes níveis) e a falta de um dispositivo de formação permanente para os ACS.

\section{A visita exploratória}

Após o retorno dos consultores do Unicef contratados para auxiliar na implantação do Pacs em Angola, eles empenharam-se em identificar parcerias para a continuidade do programa. Assim, foram contatados profissionais da UFRGS, da UFC, da Escola de Formação em Saúde da Família Visconde de Saboia e da EPSJV/Fiocruz, que se reuniram para discutir as possibilidades de cooperação técnica e apoio ao Pacs Luanda.

Como produtos dessa articulação, foram elaborados dois projetos: um de visita exploratória aprovado pelo CNPq, intitulado "Agente Comunitário de Saúde Angolano: análise das concepções e práticas em saúde e a forma- 
ção profissional", e outro de avaliação do Pacs de Luanda, denominado “Desenvolvendo Serviços de Atenção Primária à Saúde em Angola: um projeto de cooperação com o Brasil". Este relato discute o trabalho de campo realizado na primeira viagem do projeto de visita exploratória, em 2009, e teve como objetivo analisar as percepções e práticas dos ACS angolanos em relação à saúde da população, com vistas a subsidiar a construção de propostas de formação profissional.

Este trabalho faz parte de um projeto de cooperação técnica para formação profissional de ACS aprovado pelo CNPq em 2008, co-financiado pela Opas, reunindo pesquisadores brasileiros, consultores do Unicef e profissionais da saúde angolanos.

Para atingir os objetivos previstos, foi adotada como metodologia a pesquisa qualitativa, realizando entrevistas semiestruturadas com coordenadores municipais do Pacs e grupos focais com ACS nos seis municípios onde existe o Pacs implantado. Eventualmente, dependendo do contexto do município, também foram realizadas entrevistas individuais com roteiro semiestruturado com ACS, coordenadores do Pacs da DPS de Luanda ou coordenadores de bairro. Foram entrevistados seis coordenadores municipais de ACS, um coordenador de bairro, um coordenador do Pacs da Direção Provincial de Luanda e um ACS. Aconteceram, também, seis grupos focais com ACS.

\section{Percepção e práticas de saúde dos ACS angolanos}

A partir dos seis grupos focais realizados nos municípios, pretendia-se compreender a percepção dos ACS sobre seu papel, sua rotina de trabalho, suas dificuldades e seus desafios da prática, além de discutir como tem sido o processo formativo dos mesmos.

Assim como em estudos brasileiros (Bornstein e Stotz, 2008-2009), observamos que os ACS em Angola se percebem como um 'elo' entre a comunidade e os serviços de saúde. Nos grupos, foi destacado que o papel do ACS é ser um educador em saúde. Porém, muitas vezes vimos que a ênfase se dava em uma perspectiva educativa que busca convencer a população a adotar comportamentos considerados corretos pelos profissionais da saúde. Alguns ACS falaram muito no papel de "transmitir" conhecimento para a população e de mudar "comportamentos negativos" para "comportamentos positivos". Bornstein e Stotz (2008-2009) apontam que essa perspectiva educativa reforça o entendimento de que o conhecimento científico - e, portanto, o conhecimento biomédico - é aquele que é objetivo e capaz de refletir a realidade de maneira mais fidedigna e, nesse sentido, a mediação educativa realizada pelos trabalhadores da saúde se processa de forma mais autoritária. 
A pouca valorização do conhecimento popular, do senso comum, pode indicar um caminho que não favorece o fortalecimento da autonomia dos sujeitos.

Diante da situação do sistema de saúde angolano, também foi destacada a importância de o ACS estimular e facilitar o acesso, principalmente para grávidas e crianças menores de 2 anos, ao serviço de saúde. Existe um discurso forte de 'levar' as gestantes e crianças para o posto de saúde, que visa a ampliar o acesso aos serviços de saúde. Porém, consideramos que nesse processo pode haver uma 'medicalização' dos cuidados, pois os cuidados tradicionais são secundarizados.

Nos grupos realizados, os cuidados e as práticas de saúde tradicionais foram muitas vezes qualificados como 'ultrapassados', em contraposição aos cuidados oferecidos nos centros de saúde e hospitais, que representam a 'modernidade'. Pode-se entender esse movimento no contexto angolano devido à altíssima mortalidade que se dá por falta de acesso e adequação dos serviços de saúde, bem como pela escassez de profissionais devidamente qualificados para responder às necessidades em saúde da população. Assim, por um lado, pode-se justificar que o movimento rumo a melhores condições de saúde esteja concentrado em ações que favoreçam o acesso ao serviço de saúde e valorizem o cuidado praticado por um profissional dessa área.

Por outro lado, consideramos que faz parte do movimento de hegemonia da biomedicina a desvalorização e desqualificação dos demais sistemas terapêuticos. Dessa maneira, nas relações assimétricas entre esses diferentes saberes e práticas de saúde, as medicinas tradicionais são subjugadas e reprimidas (Menéndez, 2003).

Como no Brasil, o ACS angolano é morador da comunidade onde trabalha, é escolhido pela comunidade e percebemos nele um forte senso de responsabilidade e compromisso com a melhoria das condições de vida e da situação de saúde da população acompanhada. Alguns ACS também mencionaram que, quando estão trabalhando para a melhoria da comunidade, isso envolve a luta por melhores condições de vida e de saúde para a sua própria família.

Em relação às atividades desenvolvidas pelos ACS angolanos, destacouse o trabalho inicial de mapeamento e cadastramento das famílias, além do posterior acompanhamento das famílias. A principal ênfase é feita em relação às grávidas, aos recém-nascidos e à prevenção do paludismo (malária) dessa população por meio da distribuição de mosquiteiros. Outra importante ação é a distribuição de hipoclorito a fim de purificar a água, que, no entanto, é irregular pela falta de fornecimento dele pelos municípios. Já a distribuição de mosquiteiros também sofre uma forte oscilação, dependendo do fornecimento que chega aos municípios.

A carga horária de trabalho do ACS angolano foi prevista para oito horas semanais, sendo de cumprimento livre, de acordo com sua disponibilidade. Nesse ponto, é importante destacarmos que os agentes angolanos não têm 
vínculo empregatício formal com qualquer instância governamental. Eles são selecionados e recebem um subsídio, que equivale a um incentivo financeiro para o trabalho, conforme destacado anteriormente.

Entretanto, no contexto atual de desemprego da maioria da população, onde grande parte trabalha no setor informal, esse incentivo se torna uma importante fonte de renda. Ainda assim, muitos agentes têm outras atividades para garantir sua subsistência, seja de estudo, trabalho informal ou formal. Ficou claro, a partir das discussões nos grupos, que ser ACS é apenas uma das atividades desenvolvidas por essas pessoas, sendo que, diante do atraso no pagamento dos subsídios e da luta pela subsistência, essa dedicação de tempo de serviço no Pacs por vezes fica muito comprometida. O atraso dos subsídios foi a principal queixa dos ACS, sendo apontado como fator determinante para a desmotivação dos agentes, levando à desistência do trabalho por alguns deles.

Diante dessas condições, as rotinas de trabalho se mostraram muito heterogêneas: alguns dedicam um certo número de horas diárias; outros, somente os finais de semana. Ademais, para os agentes que atuam em área rural, as distâncias entre as casas representa outra dificuldade, pois eles não dispõem de qualquer meio de transporte ou auxílio financeiro para tal. A diretriz do programa prevê o acompanhamento de cem famílias, com a visita mensal das mesmas, mas foi possível constatar que essa proposta dificilmente é cumprida pela maioria dos ACS.

Mesmo com essas dificuldades, os ACS criam diferentes estratégias de trabalho com a comunidade, como mutirões de limpeza, palestras sobre diversos temas e jornal mural (exposição de fotos retratando o trabalho dos ACS na comunidade). Nas atividades de visita de campo com os agentes, percebemos um grande conhecimento das áreas e das famílias. Entretanto, algumas vezes observamos na fala dos agentes uma postura de 'vigilância' em relação às famílias para o cumprimento de orientações, como o uso de mosquiteiro e a utilização do hipoclorito. Os ACS referiram averiguar nos domicílios se os mesmos são efetivamente usados.

Nas discussões dos grupos focais, os ACS levantaram algumas dificuldades para a realização de seu trabalho, principalmente em relação à falta de materiais, como uniforme, botas, máscara, hipoclorito e mosquiteiro. A falta de hipoclorito e mosquiteiro gera um constrangimento para o ACS, pois, no início de suas atividades, foi colocado para a população que estes seriam distribuídos, o que afeta a credibilidade de seu trabalho segundo eles próprios afirmam.

Outra dificuldade que compromete a credibilidade e a inserção do ACS parece ser a relação com os serviços de saúde. Os ACS queixaram-se de que não são respeitados e reconhecidos pelos profissionais desses serviços e não possuem uma identificação que permita o livre trânsito nos serviços de saúde. Comentam que seus encaminhamentos e percepções sobre os pacientes não 
são escutados. É importante esclarecer que essa queixa ocorreu em alguns municípios, enquanto outros referiram que existe um contato com os serviços, com boa recepção dos ACS, intermediado pelo coordenador de ACS. De qualquer forma, esse foi um ponto sempre presente nos debates, pois a relação com os serviços é um dos papéis de destaque para o ACS. Esse é um ponto que suscita a discussão sobre sua função de 'elo de ligação' entre a comunidade e o serviço de saúde.

Os ACS têm também como responsabilidade o preenchimento de fichas sobre as famílias acompanhadas e as atividades desenvolvidas. A maioria deles refere não ter dificuldades no preenchimento delas, mas percebemos que os dados consolidados apresentam muitas inconsistências e falhas. Assim, a produção da informação do Pacs é outro desafio.

Apesar das dificuldades apontadas, os agentes relatam, de forma praticamente unânime, que percebem melhorias das condições de vida da população, como diminuição da mortalidade infantil e acompanhamento das gestantes, o que nos pareceu de difícil aferição diante da fragilidade dos dados disponíveis.

Em relação à formação, os ACS referiram ter participado de uma formação inicial para desenvolver as suas atividades, cujo tempo de duração variou. Nessa formação, relataram que foram discutidos temas como o preenchimento dos relatórios ou fichas de avaliação, cadastramento, controle do peso, orientação da gestante, visita domiciliar, como lidar com a comunidade e prevenção de cólera e malária. Apontaram que essa formação foi adequada para iniciar os trabalhos, mas que necessitam de reforços ou 'refrescamentos' e continuidade da formação. Quando questionados sobre que tipo de conhecimentos gostariam de ter, muitos referiram desejar ter acesso ao saber do campo biomédico, como primeiros socorros, identificação de algumas doenças e realização de procedimentos de enfermagem (curativo, vacinação). Alguns poucos ACS foram mais contundentes em demandar formação técnica na área da saúde e o reconhecimento como profissional.

\section{Desafios para a atenção primária e a formação profissional em saúde}

Durante a visita exploratória, também foi possível nos reunirmos com a direção pedagógica da Escola Técnica Profissional de Luanda e com a coordenação do departamento de Recursos Humanos da Direção Provincial de Luanda. Conversamos sobre as prioridades de formação de profissionais da DPS, a organização da formação de técnicos em Luanda e o Pacs. Sobre esse programa, fomos esclarecidos que, devido ao fato de os ACS não fazerem parte do quadro de funcionários da DPS ou do Ministério da Saúde (Minsa), de Angola, o departamento de Recursos Humanos não se responsabiliza pela formação desses trabalhadores. No entanto, pode-se destacar que, durante o Conselho 
Consultivo, que é a instância de deliberação do sistema de saúde de Angola, foi discutida a importância do Pacs em Luanda, a necessidade de aumento do subsídio dos ACS e a incorporação destes aos quadros do Minsa ou da DPS.

Pensamos que a valorização da formação desses trabalhadores está certamente relacionada com a sua institucionalização. No momento da visita, os ACS ainda estavam em uma situação precária de vinculação, recebendo subsídios atrasados, sendo que estes são entendidos como estímulo e não remuneração de trabalho. Seu compromisso de dedicação ao trabalho era de oito horas semanais e a formação estava sendo feita de maneira informal e em serviço, irregularmente, sem articulação com as instituições de ensino locais. Essas características de trabalho e formação do ACS angolano apontam para problemas na qualificação profissional desses trabalhadores, situação que também ocorre no Brasil (Morosini, 2010). Essa institucionalização está vinculada diretamente ao avanço da reconstrução e reorganização do sistema de saúde angolano e na vinculação do Pacs a essa estrutura, o que daria maior legitimidade ao trabalho dos ACS.

Ademais, ressaltamos outro desafio fundamental na implantação do Pacs em Angola, que é a adequação do programa e do trabalho do ACS às características socioculturais desse país. Afinal, como comentamos anteriormente, nos territórios visitados já identificamos diversas especificidades locais que devem ser consideradas na implantação do programa, como a estrutura de liderança tradicional (soba), a existências de práticas terapêuticas locais, a diversidade de representações de saúde-doença, o multilinguismo, entre outras. Esses e outros aspectos socioculturais devem ser reconhecidos e considerados na organização das ações de saúde e no trabalho do ACS.

Consideramos que a visita possibilitou o levantamento de dados consistentes para a discussão de projetos relacionados à formação dos ACS e coordenadores de ACS no futuro, tendo sido estabelecida uma base para o diálogo a respeito das possibilidades de cooperação técnica.

\section{Nota do Editor}

Este artigo resulta do projeto de visita exploratória, aprovado e financiado pelo CNPq, intitulado “Agente Comunitário de Saúde Angolano: análise das concepções e práticas em saúde e a formação profissional". As atividades de campo também foram co-financiadas pela Opas. Esse projeto não foi encaminhado aos comitês de ética em pesquisa das instituições brasileiras, pois era uma visita exploratória. 


\section{Notas}

1 Médica, professora-pesquisadora do Laboratório de Educação Profissional em Atenção à Saúde da Escola Politécnica de Saúde Joaquim Venâncio, Fundação Oswaldo Cruz (Laborat/EPSJV/Fiocruz), Rio de Janeiro, Brasil. Doutoranda em Saúde Pública pela Escola Nacional de Saúde Pública Sergio Arouca, Fundação Oswaldo Cruz (Ensp/Fiocruz). <analupontes@fiocruz.br>

Correspondência: Laboratório de Educação Profissional em Atenção à Saúde da Escola Politécnica de Saúde Joaquim Venâncio, Fundação Oswaldo Cruz (Laborat/EPSJV/Fiocruz), Av. Brasil, 4.365, CEP 21040-360, Manguinhos, Rio de Janeiro-RJ, Brasil.

2 Assistente social, professora-pesquisadora do Laboratório de Educação Profissional em Atenção à Saúde da Escola Politécnica de Saúde Joaquim Venâncio, Fundação Oswaldo Cruz (Laborat/EPSJV/Fiocruz), Rio de Janeiro, Brasil. Doutora em Saúde Pública pela Escola Nacional de Saúde Pública Sergio Arouca, Fundação Oswaldo Cruz (Ensp/Fiocruz). $<$ vejoana@fiocruz.br>

3 Médica, professora do Departamento de Medicina Social da Universidade Federal do Rio Grande do Sul (UFRGS), Porto Alegre, Rio Grande do Sul, Brasil. Doutora em Epidemiologia pela Universidade Federal do Rio Grande do Sul (UFRGS). <giugli@hotmail.com>

4 O coordenador de bairro é uma liderança eleita pela comunidade, mas subordinada a uma liderança tradicional, o soba.

\section{Referências}

AQUINO, Rosana; OLIVEIRA, Nelson F. de; BARRETO Maurício L. Impact of the family health program on infant mortality in Brazilian municipalities. American Journal of Public Health, Washington, DC, v. 99, n. 1, p. 87-93, jan. 2009.

BORNSTEIN, Vera Joana; STOTZ, Eduardo Navarro. O trabalho dos agentes comunitários de saúde: entre a mediação convencedora e a transformadora. Trabalho, Educação e Saúde, Rio de Janeiro, v. 6, n. 3, p. 457480, nov./fev. 2008-2009.

MACINKO, James; GUANAIS, Frederico C.; SOUZA, Maria de Fátima M. de. Evaluation of the impact of the Family Health Program on infant mortality in Brazil, 1990-2002.
Journal of Epidemiology and Community Health, v. 60, n. 1, p. 13-19, jan. 2006.

MACINKO, James et al. Going to scale with community-based primary care: an analysis of the family health program and infant mortality in Brazil, 1999-2004. Social Science \& Medicine, v. 65, n. 10, p. 2070-2080, nov. 2007.

MENÉNDEZ, Eduardo L. Modelos de atención de los padecimientos: de exclusiones teóricas y articulaciones prácticas. Ciência \& Saúde Coletiva, Rio de Janeiro, v. 8, n. 1, p. 185-207, 2003.

MOROSINI, Márcia V. Educação e trabalho em disputa no SUS: a política de formação 
dos agentes comunitário de saúde. Rio de Janeiro: EPSJV, 2010.

OLIVEIRA, Miguel dos Santos e ARTMANN, Elizabeth. Regionalização dos serviços de saúde: desafios para o caso de Angola. Cadernos de Saúde Pública, Rio de Janeiro, v. 25, n. 4, p. 751-760, abr. 2009.

SANTOS, Daniel. Economia, democracia e justiça em Angola: o efêmero e o permanente. Estudos Afro-Asiáticos, Rio de Janeiro, v. 23, n. 1, p. 99-133, 2001.
SVITONE, Ennio Cufino et al. Primary health care lessons from the Northeast of Brazil: the Agentes de Saúde Program. Pan American Journal of Public Health, v. 7, n. 5, p. 293-301, 2000.

Recebido em 21/09/2010

Aprovado em 01/07/2011 